\title{
Clinical characteristics and outcomes in acute myocardial infarction patients with versus without any cardiovascular risk factors
}

\author{
Ah-Ra Choi ${ }^{1}$, Myung Ho Jeong ${ }^{1}$, Young Joon Hong ${ }^{1}$, Seok-Joon Sohn ${ }^{2}$, Hyun Yi Kook ${ }^{1}$, Doo Sun Sim ${ }^{1}$, \\ Young Keun Ahn ${ }^{1}$, Ki Hong Lee ${ }^{1}$, Jae Yeong Cho ${ }^{1}$, Young Jo Kim³, Myeong Chan Cho ${ }^{4}$, Chong Jin Kim \\ and other Korea Acute Myocardial Infarction Registry Investigators
}

\author{
${ }^{1}$ The Heart Center of Chonnam \\ National University Hospital, \\ Gwangju; ${ }^{2}$ Department of Preventive \\ Medicine, Chonnam National \\ University Medical School, Gwangju; \\ ${ }^{3}$ Department of Cardiology, \\ Yeungnam University Medical \\ Center, Daegu; ${ }^{4}$ Department of \\ Cardiology, Chungbuk National \\ University Hospital, Cheongju; \\ ${ }^{5}$ Department of Cardiology, Kyung \\ Hee University Hospital, Seoul, \\ Korea
}

Received: February 9, 2018

Revised : April 6, 2018

Accepted: April 12, 2018

\section{Correspondence to}

Myung Ho Jeong, M.D.

The Heart Center of Chonnam

National University Hospital, 42

Jebong-ro, Dong-gu, Gwangju

61469 , Korea

Tel: $+82-62-220-6243$

Fax: +82-62-228-7174

E-mail: myungho@chollian.net
Background/Aims: Although cardiovascular (CV) risk factors are well established, some patients experience acute myocardial infarction (AMI) even without any risk factors.

Methods: We analyzed total 11,390 patients (63.6 \pm 12.6 years old, 8,401 males) with AMI enrolled in Korea Acute Myocardial Infarction Registry-National Institute of Health from November, 2011 to December, 2015. Patients were divided into two groups according to the presence of any CV risk factors (group I, without risk factors, $\mathrm{n}=1,420$ [12.5\%]; group II, with risk factors, $\mathrm{n}=9,970$ [87.5\%]). In-hospital outcomes were defined as in-hospital mortality and complications. One-year clinical outcomes were defined as the composite of major adverse cardiac events (MACE).

Results: Group I was older $(67.3 \pm 11.6$ years old vs. $63.0 \pm 12.7$ years old, $p<0.001)$ and had higher prevalence of female gender ( $36.2 \%$ vs. $24.8 \%, p<0.001)$ than the group II. Group I experienced less previous history of angina pectoris (7.0\% vs. $9.4 \%, p=0.003)$ and the previous history of cerebrovascular accidents $(3.4 \%$ vs. $6.9 \%, p<0.001)$. In-hospital mortality ( $2.6 \%$ vs. $3.0 \%, p=0.450$ ) and complications (20.6\% vs. $20.0 \%, p=0.647$ ) were no differences between the groups. And 1 year clinical outcomes ( $5.7 \%$ vs. $5.1 \%, p=0.337$ ) were no differences between the groups. In multivariate logistic regression analysis, serum creatinine level (hazard ratio, 1.35; $95 \%$ confidence interval, 1.05 to $1.75 ; p=0.021$ ) were independent predictors of 1 year MACE in patients without any CV risk factors.

Conclusions: Elderly female patients were prone to develop AMI even without any modifiable CV risk factors. We suggest that more intensive care is needed in AMI patients without any CV risk factors who have high serum creatinine levels.

Keywords: Risk factors; Myocardial infarction; Prognosis

\section{INTRODUCTION}

Worldwide, cardiovascular (CV) disease is a leading cause of death. With an extension of life expectancy and a changing of diet, the incidence of acute myocardial infarction (AMI) is increasing and becoming a major cause of morbidity and mortality in Korea [1].

There are many known CV risk factors [2-7]. Some of 
the known CV risk factors globally are; old age, high blood pressure, sociodemographic, economical transition, family history of coronary heart disease, increased cigarette smoking, alcohol intake, high serum lipid, physical inactivity, obesity, diabetes, and poor nutrition [3-7]. Apart from non-modifiable risk factors such as age, male, and genetic predisposition, many other risk factors could be controlled by changes to individual life style or medical therapy [2]. Of these, the major modifiable CV risk factors are considered obesity, smoking, hypertension, diabetes mellitus, and dyslipidemia [2]. In this way, although CV risk factors are well established, some patients experience AMI even without any risk factors. But there was little known about the patients without any risk factors.

Previous most studies have concentrated the prevalence of CV risk factors in patients with AMI. There are few data available on the clinical characteristics and outcomes in the AMI patients without CV risk factors. In this study we analyzed to determine the incidence, clinical characteristics, and outcomes in AMI patients with versus without any major modifiable CV risk factors. Additionally, we analyzed the association between the hospital discharge risk model in the previous study for Korean AMI patients [8] and outcomes in AMI with versus without any major modifiable CV risk factors.

\section{METHODS}

\section{Study design and population}

This study population was selected for AMI patients who were enrolled in Korea Acute Myocardial Infarction Registry-National Institute of Health (KAMIR-NIH). KAMIR-NIH registry is a prospective, multicenter, webbased observational cohort study, designed to collect data on baseline characteristics, real-world treatment practice and outcomes of the AMI patients diagnosed from 20 university hospitals with facilities for primary percutaneous coronary intervention (PCI) from November 2011 to December 2015 in Korea [1]. This protocol approved by Institutional Review Board (CNUH-2011172) of each investigating hospital and informed consent was obtained from all patients [1].

A total of 13,106 AMI patients who enrolled KAMIRNIH from November 2011 to December 2015. Among

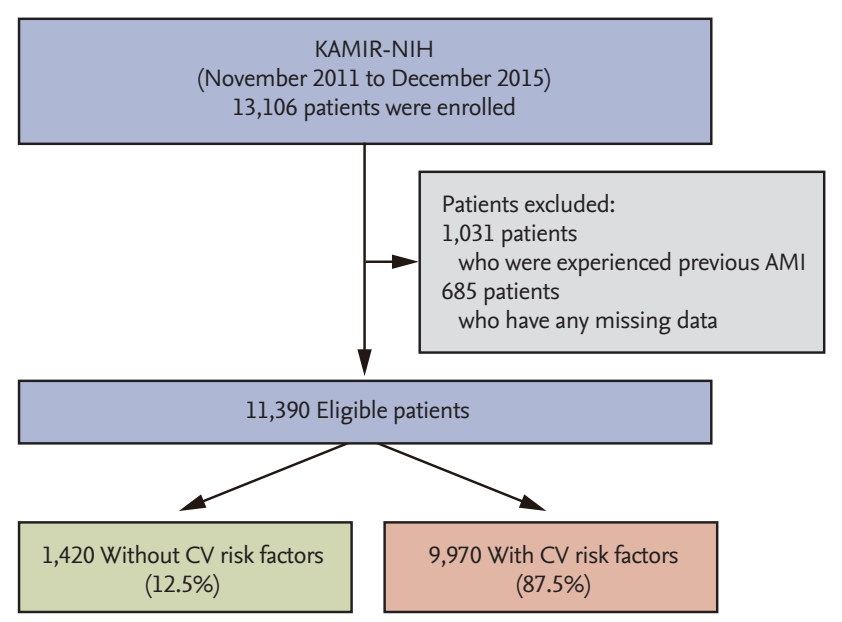

Figure 1. Study flow chart. KAMIR-NIH, Korea Acute Myocardial Infarction Registry-National Institute of Health; AMI, acute myocardial infarction; CV, cardiovascular.

these, the we analyzed the eligible 11,390 patients $(63.6 \pm$ 12.6 years old, male $73.8 \%$ ) excluded 1,031 patients who were experienced previous AMI and 685 patients who have any missing data. Patients were divided into two groups according to the presence of any major modifiable $\mathrm{CV}$ risk factors. Patients without any CV risk factors were $1,420(67.3 \pm 11.6$ years old, male $63.8 \%)$ and patients with any $\mathrm{CV}$ risk factors were $9,970(63.0 \pm 12.7$ years old, male 73.8\%) (Fig. 1).

\section{Definition and clinical endpoint}

AMI was defined as cardiomyocyte necrosis in a clinical setting consistent with acute myocardial ischemia. AMI was diagnosed by the characteristics presentation, serial changes on 12-lead electrocardiographic suggesting infarction, and arise in cardiac markers, preferably cardiac troponins, with at least one value above the 99th percentile of the upper reference limit [9].

$\mathrm{CV}$ risk factors were defined as smoking, obesity, hypertension, diabetes mellitus, and dyslipidemia. Prevalence of CV risk factors was as follow: (1) smoking was current smoking; (2) obesity was body mass index $\geq 25$ $\mathrm{kg} / \mathrm{m}^{2}$; (3) hypertension was history of hypertension with medical treatment; (4) diabetes mellitus was history of diabetes mellitus with medication or using insulin; and (5) dyslipidemia was history of dyslipidemia with medical treatment or a fasting total cholesterol level $\geq 200 \mathrm{mg} / \mathrm{dL}$. 
Clinical characteristics were collected in the index hospitalization. Clinical endpoint was the composite of major adverse cardiac events (MACE) during 12 months of clinical follow-up. MACE was defined as all cause death, non-fatal myocardial infarction, repeated PCI, coronary artery bypass graft, cerebrovascular event, all cause re-hospitalization, and stent thrombosis.

\section{Statistical analysis}

The software package SPSS version 23.0 for Windows (IBM Co., Armonk, NY, USA) was used for all statistical analyses. Continuous variables were evaluated as mean \pm standard deviation. Categorical variables were evaluated as counts or percentages as appropriate. Differences between groups were performed using the chisquare test or Fisher's exact test. Kaplan-Meier curves were compared for all cause death and MACE, and the log rank test was used to test the differences in the survival curves. Multivariate analysis was used to determine that the association between the hospital discharge risk model and MACE. And the constructed six independent variables (age, Killip class, serum creati-

Table 1. Baseline clinical characteristics in patients with versus without cardiovascular risk factors

\begin{tabular}{|c|c|c|c|}
\hline Variable & Without risk factors $(n=1,420)$ & With risk factors $(\mathrm{n}=9,970)$ & $p$ value \\
\hline Age, yr & $67.3 \pm 11.6$ & $63.0 \pm 12.7$ & $<0.001$ \\
\hline \multicolumn{4}{|l|}{ Sex } \\
\hline Male & $906(63.8)$ & $7,495(75 \cdot 2)$ & $<0.001$ \\
\hline Female & $514(36.2)$ & $2,475(24.8)$ & $<0.001$ \\
\hline Symptom & & & 0.044 \\
\hline Atypical pain & $201(14.2)$ & $1,247(12.5)$ & \\
\hline Typical pain & $1,219(85.8)$ & $8,723(87.5)$ & \\
\hline First medical contact & & & 0.086 \\
\hline PCI center & $460(32.4)$ & $3,128(31.4)$ & \\
\hline Non-PCI center & $783(55.1)$ & $5,380(54 \cdot 0)$ & \\
\hline EMS & $177(10.8)$ & $1,462(14 \cdot 7)$ & \\
\hline \multicolumn{4}{|l|}{ Previous history } \\
\hline Angina & $99(7.0)$ & $942(9.4)$ & 0.003 \\
\hline $\mathrm{HF}$ & $17(1.2)$ & $127(1.3)$ & 0.887 \\
\hline CVA & $48(3 \cdot 4)$ & $690(6.9)$ & $<0.001$ \\
\hline Family history of CAD & $78(5 \cdot 5)$ & $656(6.6)$ & 0.135 \\
\hline \multicolumn{4}{|l|}{ Physical finding } \\
\hline $\mathrm{SBP}, \mathrm{mmHg}$ & $128.1 \pm 29.6$ & $131.3 \pm 29.4$ & $<0.001$ \\
\hline $\mathrm{DBP}, \mathrm{mmHg}$ & $77.8 \pm 18.2$ & $79 \cdot 3 \pm 17 \cdot 9$ & 0.003 \\
\hline Heart rate, beats/min & $76.2 \pm 18.7$ & $78.8 \pm 19.1$ & $<0.001$ \\
\hline Killip class & & & 0.676 \\
\hline I & $1,121(78.9)$ & $7,920(79.4)$ & \\
\hline II-IV & $299(21.1)$ & $2,050(20.6)$ & \\
\hline Final diagnosis & & & 0.650 \\
\hline NSTEMI & $718(50.6)$ & $5,109(51.2)$ & \\
\hline STEMI & $702(49.4)$ & $4,861(48.8)$ & \\
\hline
\end{tabular}

Values are presented as mean $\pm \mathrm{SD}$ or number $(\%)$.

PCI, percutaneous coronary intervention; EMS, emergency medical service; HF, heart failure; CVA, cerebrovascular accident; CAD, coronary artery disease; SBP, systolic blood pressure; DBP, diastolic blood pressure; NSTEMI, non-ST segment elevation myocardial infarction; STEMI, ST segment elevation myocardial infarction. 
Table 2. Baseline echocardiographic, laboratory and coronary, angiographic findings in patients with versus without cardiovascular risk factors

\begin{tabular}{|c|c|c|c|}
\hline Variable & Without risk factors $(n=1,420)$ & With risk factors $(\mathrm{n}=9,970)$ & $p$ value \\
\hline \multicolumn{4}{|l|}{ Echocardiographic finding } \\
\hline LVEF, \% & $52.2 \pm 10.7$ & $52.4 \pm 11.1$ & 0.519 \\
\hline $\mathrm{LVEF}<40 \%$ & $162(11.4)$ & $1,135(11.4)$ & 0.968 \\
\hline \multicolumn{4}{|l|}{ Laboratory finding } \\
\hline Admission glucose, mg/dL & $145.2 \pm 54.4$ & $171.2 \pm 82.5$ & $<0.001$ \\
\hline Admission creatinine, $\mathrm{mg} / \mathrm{dL}$ & $0.9 \pm 0.5$ & $1.1 \pm 1.2$ & $<0.001$ \\
\hline Peak CK, U/L & $1,071.5 \pm 2,842.4$ & $1,016.4 \pm 1,742.7$ & 0.349 \\
\hline Peak CK-MB, ng/mL & $113.7 \pm 140.5$ & $113.0 \pm 170.3$ & 0.889 \\
\hline Peak troponin I, ng/mL & $46.7 \pm 136.4$ & $47.7 \pm 100.9$ & 0.773 \\
\hline Total cholesterol, mg/dL & $184.0 \pm 44 \cdot 3$ & $179.8 \pm 46.4$ & 0.002 \\
\hline Triglyceride, mg/dL & $104.9 \pm 88.4$ & $139.7 \pm 126.6$ & $<0.001$ \\
\hline HDL-C, mg/dL & $46.5 \pm 13.6$ & $42.5 \pm 12.2$ & $<0.001$ \\
\hline LDL-C, mg/dL & $118.6 \pm 41.7$ & $113.2 \pm 39.7$ & $<0.001$ \\
\hline NT-proBNP, pg/mL & $2,447 \cdot 5 \pm 8,523 \cdot 7$ & $2,496.5 \pm 8,198.7$ & 0.867 \\
\hline hs-CRP, mg/dL & $1.21 \pm 3.4$ & $1.53 \pm 6.2$ & 0.141 \\
\hline \multicolumn{4}{|l|}{ Coronary angiographic finding } \\
\hline PCI & $1,276(89.9)$ & $9,058(90.9)$ & 0.123 \\
\hline Successful PCI & $1,263(99.0)$ & $8,960(98.9)$ & 0.962 \\
\hline No. of involved vessels & & & $<0.001$ \\
\hline Single vessel & $725(51.1)$ & $4,597(46.1)$ & \\
\hline Left main or multivessel & $607(42.7)$ & $4,929(49 \cdot 4)$ & \\
\hline Target lesion vessel & & & 0.003 \\
\hline Left anterior descending & $669(47.1)$ & $4,214(42.3)$ & \\
\hline Left circumflex & $204(14.4)$ & $1,605(16.1)$ & \\
\hline Right coronary & $382(26.9)$ & $3,437(30.2)$ & \\
\hline Left main & $26(1.8)$ & $197(2.0)$ & \\
\hline ACC/AHA type & & & 0.789 \\
\hline Type A/B1 & $166(13.0)$ & $1,370(13.2)$ & \\
\hline Type B2/C & $1,114(87.0)$ & $8,980(86.8)$ & \\
\hline
\end{tabular}

Values are presented as mean \pm SD or number (\%).

LVEF, left ventricular ejection fraction; CK, creatine kinase; HDL-C, high density lipoprotein cholesterol; LDL-C, low density lipoprotein cholesterol; NT-proBNP, N-terminal pro B-type natriuretic peptide; hs-CRP, high-sensitivity C-reactive protein; PCI, percutaneous coronary intervention; ACC/AHA, American College of Cardiology/American Heart Association.

nine, no in-hospital PCI, left ventricular ejection fraction [LVEF], and admission glucose) in the previous study were used to the prognostic significance [8]. The all statistically analyses were 2-tailed, with statistical significance defined as value of $p<0.05$.

\section{RESULTS}

\section{Baseline clinical characteristics}

The baseline clinical characteristics are listed in Table 1. The group without risk factors was older $(p<0.001)$ and had higher prevalence of female gender $(p<0.001)$ than the group with risk factors. Although most of the pa- 
Table 3. In-hospital clinical outcomes in patients with versus without cardiovascular risk factors

\begin{tabular}{|c|c|c|c|}
\hline Variable & Without risk factors $(n=1,420)$ & With risk factors $(\mathrm{n}=9,970)$ & $p$ value \\
\hline In-hospital mortality & $37(2.6)$ & $302(3.0)$ & 0.450 \\
\hline Cardiac death & $30(2.1)$ & $242(2.4)$ & 0.512 \\
\hline Non-cardiac death & $7(0.5)$ & $60(0.6)$ & 0.852 \\
\hline In-hospital complications & $292(20.6)$ & $1,997(20.0)$ & 0.647 \\
\hline Cardiogenic shock & $135(9.5)$ & $818(8.2)$ & 0.099 \\
\hline Newly developed HF & $59(4.2)$ & $424(4 \cdot 3)$ & 0.938 \\
\hline Recurrent ischemia & $11(0.8)$ & $93(0.9)$ & 0.656 \\
\hline Recurrent infarction & $3(0.2)$ & $40(0.4)$ & 0.359 \\
\hline Stent thrombosis & $3(0.2)$ & $35(0.4)$ & 0.620 \\
\hline Cerebrovascular event & $8(0.6)$ & $64(0.6)$ & 0.859 \\
\hline Major bleeding & $24(1.7)$ & $206(2.1)$ & 0.417 \\
\hline Minor bleeding & $50(3.5)$ & $317(3.2)$ & 0.475 \\
\hline AV block & $27(1.9)$ & $233(2.3)$ & 0.342 \\
\hline $\mathrm{VT}$ & $40(2.8)$ & $342(3.4)$ & 0.271 \\
\hline VF & $32(2.3)$ & $163(1.6)$ & 0.096 \\
\hline Atrial fibrillation & $53(3.7)$ & $316(3.2)$ & 0.262 \\
\hline Acute kidney injury & $6(0.4)$ & $86(0.9)$ & 0.110 \\
\hline Sepsis & $8(0.6)$ & $60(0.6)$ & 1.000 \\
\hline Multiorgan failure & $7(0.5)$ & $63(0.6)$ & 0.716 \\
\hline
\end{tabular}

Values are presented as number (\%).

HF, heart failure; AV, atrioventricular; VT, ventricular tachycardia; VF, ventricular fibrillation.

tients without risk factors (85.8\%) complained of typical pain, only $10.8 \%$ utilized the emergency medical service for first medical contact. The group without risk factors experienced less previous history of angina pectoris $(p=$ 0.003 ) and the previous history of cerebrovascular accident $(p<0.001)$. And the previous history of heart failure (HF, $p=0.887)$ and family history of coronary artery disease $(p=0.135)$ were no difference in the groups. Also the Killip class $(p=0.676)$ and the final diagnosis of ST segment elevation myocardial infarction (STEMI) and non-ST segment elevation myocardial infarction (NSTEMI) $(p=0.650)$ were no difference between the groups.

\section{Baseline echocardiographic, laboratory, and coro- nary angiographic findings}

The echocardiographic, laboratory, and coronary angiographic findings are listed in Table 2. The mean of $\operatorname{LVEF}(p=0.519)$ were no difference in the groups. The level of admission glucose $(p<0.001)$, creatinine $(p<$
$0.001)$, and triglyceride $(p<0.001)$ were lower in the group without risk factors. But total cholesterol $(p=$ 0.002), high density lipoprotein cholesterol $(p<0.001)$, and low density lipoprotein cholesterol $(p<0.001)$ were higher in the group without the risk factors. And the cardiac enzyme, N-terminal pro B-type natriuretic peptide and high-sensitivity C-reactive protein were no difference between the groups. The PCI was performed in almost all patients in this study. And the rate of successful PCI was also high. Coronary angiographic findings showed the target vessel in the order of left anterior descending artery, right coronary artery, left circumflex, and left main artery in two groups. The morphology of lesion type by American College of Cardiology/American Heart Association (ACC/AHA) classification ( $p=$ 0.789) were no difference between the groups.

\section{In-hospital outcomes}

In-hospital outcomes are listed in Table 3. In-hospital mortality were no difference between the groups $(p=$ 
Table 4. One-year clinical outcomes in patients with versus without cardiovascular risk factors

\begin{tabular}{lccc}
\hline Variable & Without risk factors $(\mathrm{n}=1,420)$ & With risk factors $(\mathrm{n}=9,970)$ & $p$ value \\
\hline One-year MACE & $81(5.7)$ & $508(5.1)$ & 0.337 \\
\hline All cause death & $28(2.0)$ & $125(1.3)$ & 0.031 \\
\hline Cardiac death & $15(1.1)$ & $72(0.7)$ & 0.190 \\
Non-cardiac death & $13(0.9)$ & $53(0.5)$ & 0.090 \\
Recurrent MI & $5(0.4)$ & $60(0.6)$ & 0.344 \\
Repeated PCI & $40(2.8)$ & $271(2.7)$ & 0.802 \\
CABG & $4(0.3)$ & $11(0.1)$ & 0.107 \\
Cerebrovascular event & $4(0.3)$ & $51(0.5)$ & 0.308 \\
\hline All cause rehospitalization & $9(0.6)$ & $75(0.8)$ & 0.741 \\
Stent thrombosis & $2(0.1)$ & $8(0.1)$ & 0.360 \\
\hline
\end{tabular}

Values are presented as number (\%).

MACE, major adverse cardiac event; MI, myocardial infarction; PCI, percutaneous coronary intervention; CABG, coronary artery bypass graft.

0.450). Also in-hospital complications included cardiogenic shock, newly developed HF, recurrent ischemia, recurrent infarction, stent thrombosis, cerebrovascular event, major bleeding, minor bleeding, atrioventricular block, ventricular tachycardia, ventricular fibrillation, atrial fibrillation, acute kidney injury, sepsis, and multiorgan failure were no difference between the groups $(p=$ 0.647).

\section{One-year clinical outcomes}

One-year clinical outcomes are listed in Table 4. The rate of all cause death was higher in the group without risk factors $(p=0.031)$. But the composite of MACE were no difference between the groups $(p=0.337)$. Kaplan-Meier curves in patients with versus without CV risk factors are presented in Fig. 2. The cumulative survival freedom from all cause death $(p=0.632$, log rank test) and the cumulative freedom from MACE ( $p=0.306$, log rank test) were not significantly different between the groups.

The result of multivariate logistic analysis for association between the six independent predictors of KAMIR score and 1-year MACE is listed in Tables 5 and 6. Age, LVEF, and admission serum creatinine were independent predictors in whole study population. The age (hazard ratio [HR], 1.02; 95\% confidence interval [CI], 1.02 to 1.03; $p<0.001$ ), LVEF (HR, 1.55; 95\% CI, 1.21 to 1.98; $p=0.001$ ), and admission serum creatinine (HR, 1.10; $95 \%$ CI, 1.04 to 1.15 ; $<$ 0.001) were independent predictors in patients with CV risk factors. But the only admission serum creatinine (HR, 1.35; 95\% CI, 1.05 to 1.75; $p=0.021)$ was independent predictor in patients without CV risk factors.

\section{DISCUSSION}

The present study was presented to determine the incidence, clinical characteristics, and outcomes in first AMI patients without any CV risk factors. The CV risk factors were defined as the traditional modifiable CV risk factors. In this study, the clinical characteristics, in-hospital outcomes, complications, and 1-year MACE were not significantly different between the patients without and with modifiable CV risk factors. To our knowledge, this analysis is the largest study to investigate long term outcomes of the patients without CV risk factors.

There are few data available on the clinical characteristics and outcomes in the AMI patients without CV risk factors. The evidence is indisputable that the major risk factors are predictive of coronary heart disease occurrence [3-5]. And modification of the major risk factors has been convincingly shown to reduce the risk of future cardiac events [10].

In this study, the $12.5 \%$ of the AMI patients had no risk factors documented at initial hospitalization. Our 

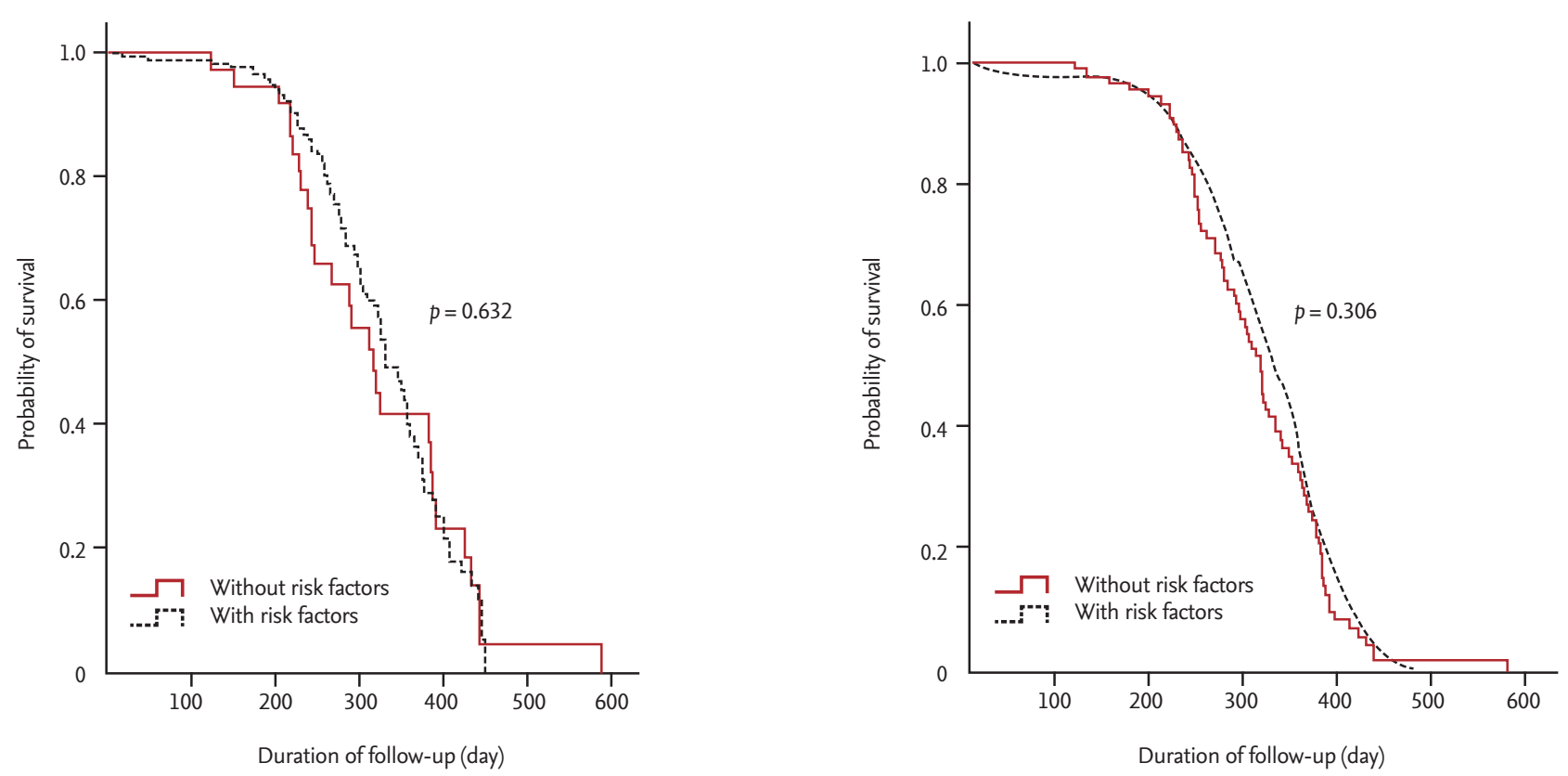

Figure 2. Kaplan-Meier curves: all cause death (A) and major adverse cardiac events (B) free survival rate in the study population.

Table 5. Multivariate analysis for association between the six independent predictors of KAMIR score and MACE in patients in whole study population

\begin{tabular}{lccr}
\hline Variable & HR & $95 \%$ CI & p value \\
\hline Age & 1.02 & $1.01-1.03$ & $<0.001$ \\
Killip class & 1.14 & $0.93-1.40$ & 0.216 \\
LVEF & 1.44 & $1.14-1.82$ & 0.002 \\
Serum creatinine & 1.10 & $1.05-1.16$ & $<0.001$ \\
Admission glucose & 1.00 & $1.00-1.00$ & 0.100 \\
No in-hospital PCI & 0.94 & $0.71-1.25$ & 0.672 \\
\hline
\end{tabular}

HR was calculated by multivariate logistic analysis.

KAMIR, Korea Acute Myocardial Infarction Registry; MACE, major adverse cardiac event; HR, hazard ratio; CI, confidence interval; LVEF, left ventricular ejection fraction; PCI, percutaneous coronary intervention.

analysis is consistent with previous studies reporting between $80 \%$ and $90 \%$ of AMI patients with at least one modifiable CV risk factors [10-14]. Nevertheless, the incidence of the AMI patients without CV risk factors is unclear. Many of these literatures were limited to specific populations and may not be generalizable to the entire AMI population. The patients without risk factors were significantly older. This result is consistent with previous studies reporting median age decreased as the number of risk factors increased [10,13,14]. This may reflect a natural survival bias because patients with risk factors die at a much younger age [15,16].

In this result showed that the patients without modifiable CV risk factors have not the favorable prognostic outcomes despite having similar clinical characteristics such as Killip classification, LVEF, and PCI rate than the patients with any CV risk factors. In-hospital mortality and complications were showed little higher trend in patients with risk factors, but there was not statistical significance. This finding is consistent with the previous studies [13,14,17]. Canto et al. [14] reported that among patients with incident AMI without prior $\mathrm{CV}$, in-hospital mortality was inversely related to the number of coronary heart disease risk factors. In one study of NSTEMI patients in the Can Rapid Risk Stratification of Unstable Angina Patients Suppress Adverse Outcomes With Early Implementation of the ACC/AHA Guidelines study (CRUSADE), they reported the mortality rates were highest in patients without risk factors, but this association was consistent and was attenuated prominently after multivariable adjustment [13]. Nevertheless little is known about the long term outcomes in the patients without CV risk factors. In this study, 1-year composite of MACE was not different between the patients without and with CV risk factors. But the 
Table 6. Multivariate analysis for association between the six independent predictors and MACE in patients with versus without cardiovascular risk factors

\begin{tabular}{|c|c|c|c|c|c|c|}
\hline \multirow{2}{*}{ Variable } & \multicolumn{3}{|c|}{ Without risk factors } & \multicolumn{3}{|c|}{ With risk factors } \\
\hline & HR & $95 \% \mathrm{CI}$ & $p$ value & HR & $95 \%$ CI & $p$ value \\
\hline Age & 1.02 & $0.99-1.04$ & 0.158 & 1.02 & $1.01-1.03$ & $<0.001$ \\
\hline Killip class & 1.24 & $0.71-2.16$ & 0.447 & 1.12 & $0.89-1.39$ & 0.334 \\
\hline LVEF & 0.76 & $0.35^{-1.61}$ & 0.459 & 1.55 & $1.21-1.98$ & 0.001 \\
\hline Serum creatinine & 1.35 & $1.05-1.75$ & 0.021 & 1.10 & $1.04-1.15$ & 0.001 \\
\hline Admission glucose & 1.00 & $1.00-1.00$ & $0.45^{8}$ & 1.00 & $1.00-1.00$ & 0.120 \\
\hline No in-hospital PCI & 0.81 & $0.36-1.80$ & 0.603 & 0.96 & $0.71-1.30$ & 0.807 \\
\hline
\end{tabular}

HR was calculated by multivariate logistic analysis.

MACE, major adverse cardiac event; HR, hazard ratio; CI, confidence interval; LVEF, left ventricular ejection fraction; PCI, percutaneous coronary intervention.

all cause death rate was higher in patients without risk factors. There are several thinkable explanations. First, it is possible that some patients without risk factors would have unaware true prevalence of the defined risk factors. In addition, the patients without risk factors may have had other risk factors that may influence MACE, such as insulin resistance, psychosocial factors, poor nutrition, physical inactivity, alcohol drinking, or non-CV comorbidities [14]. Second, the first AMI patients with CV risk factors might be received more evidence-based medications such as aspirin, statins, angiotensin II receptor blockers, and angiotensin-converting enzyme inhibitors. The patients with risk factors may have been attenuated the MACE through more intensive treatment and management before the first AMI and after AMI [14]. Third, the patients with risk factors may be more likely to have regularly met a physician as outpatients before the first AMI and after AMI. The favorable management of risk factors has been one of the major evidences of declining mortality from myocardial infarction during the past [18-20].

In this study, we analyzed to know the association between the previous six independent predictors of KAMIR score and 1-year MACE. The predictors of 1-year MACE are known old age, high Killip classification, no PCI during hospitalization, high admission creatinine, low LVEF, and high admission glucose [8]. But, the independent predictors for 1-year MACE in whole populations were old age, low LVEF, and high admission serum creatinine. But the independent predictor in patients without risk factors was only high admission serum creatinine. In routine clinical practice, together with previous data that the AMI patients with high creatinine level have a high risk of contrast induced nephropathy, ischemic and bleeding complications after PCI. Therefore, it needs to be considered observation of renal function and the use of appropriate antiplatelet agents depending on the patient's condition. And all attempts must be made to promote the use of more aggressive therapies, when they can be applied with an acceptable level of safety [21,22].

The present study has several limitations. This is non-randomized prospective registry-based study and was therefore subject to the limitations relative to this type of clinical investigation. And the defined CV risk factors were included on presence or absence of these physical features on clinical history rather than on actual measurements of blood pressure, blood glucose, or current amount of smoking. Also, the severity and number of risk factors were not considered. Nevertheless, we believe that the AMI patients without CV risk factors have not the favorable prognostic outcomes than the patients with any CV risk factors.

In conclusion, elderly female patients were prone to develop AMI even without modifiable CV risk factors. The first AMI patients without CV risk factors have not the favorable prognostic outcomes than the patients with any CV risk factors. Therefore, we suggests that more intensive care is needed in first AMI patients without CV risk factors who have a high serum creatinine levels at admission. 


\section{KEY MESSAGE}

1. The $12.5 \%$ of the acute myocardial infarction patients had no modifiable risk factors documented at initial hospitalization.

2. The patients without modifiable cardiovascular (CV) risk factors have not the favorable prognostic outcomes despite having similar clinical characteristics.

3. The only admission serum creatinine (hazard ratio, 1.35; 95\% confidence interval, 1.05 to 1.75 ; $p=0.021$ ) was independent predictor of 1 year major adverse cardiac event in patients without modifiable CV risk factors.

\section{Conflict of interest}

No potential conflict of interest relevant to this article was reported.

\section{Acknowledgments}

The authors thank all of the clinical investigators who contributed time and effort to this study, Korea Acute Myocardial Infarction Registry-National Institute of Health (KAMIR-NIH) Investigators. The KAMIR-NIH was supported by a fund (2016-ER6304-01) from Research of Korea Centers for Disease Control and Prevention, Republic of Korea.

\section{REFERENCES}

1. Kim JH, Chae SC, Oh DJ, et al. Multicenter cohort study of acute myocardial infarction in Korea: interim analysis of the Korea Acute Myocardial Infarction Registry-National Institutes of Health Registry. Circ J 2016;80:14271436.

2. Hubacek JA, Stanek V, Gebauerova M, et al. Traditional risk factors of acute coronary syndrome in four different male populations: total cholesterol value does not seem to be relevant risk factor. Physiol Res 2017;66(Supplementum 1):S121-S128.

3. Mohammadnezhad M, Mangum T, May W, Lucas JJ, Ailson S. Common modifiable and non-modifiable risk factors of cardiovascular disease (CVD) among Pacific countries. World J Cardiovasc Surg 2016;6:153-170.

4. Canto JG, Kiefe CI, Rogers WJ, et al. Atherosclerotic risk factors and their association with hospital mortality among patients with first myocardial infarction (from the National Registry of Myocardial Infarction). Am J Cardiol 2012;110:1256-1261.

5. Anand SS, Islam S, Rosengren A, et al. Risk factors for myocardial infarction in women and men: insights from the INTERHEART study. Eur Heart J 2008;29:932-940.

6. Hata J, Nagai A, Hirata M, et al. Risk prediction models for mortality in patients with cardiovascular disease: the BioBank Japan project. J Epidemiol 2017;27:S71-S76.

7. Torpy JM, Burke AE, Glass RM. JAMA patient page. Coronary heart disease risk factors. JAMA 2009;302:2388.

8. Kim HK, Jeong MH, Ahn Y, et al. Hospital discharge risk score system for the assessment of clinical outcomes in patients with acute myocardial infarction (Korea Acute Myocardial Infarction Registry [KAMIR] score). Am J Cardiol 2011;107:965-971.

9. Thygesen K, Alpert JS, Jaffe AS, et al. Third universal definition of myocardial infarction. Eur Heart J 2012;33:25512567.

10. Khot UN, Khot MB, Bajzer CT, et al. Prevalence of conventional risk factors in patients with coronary heart disease. JAMA 2003;290:898-904.

11. Yusuf S, Hawken S, Ounpuu S, et al. Effect of potentially modifiable risk factors associated with myocardial infarction in 52 countries (the INTERHEART study): case-control study. Lancet 2004;364:937-952.

12. Greenland P, Knoll MD, Stamler J, et al. Major risk factors as antecedents of fatal and nonfatal coronary heart disease events. JAMA 2003;290:891-897.

13. Roe MT, Halabi AR, Mehta RH, et al. Documented traditional cardiovascular risk factors and mortality in nonST-segment elevation myocardial infarction. Am Heart J 2007;153:507-514.

14. Canto JG, Kiefe CI, Rogers WJ, et al. Number of coronary heart disease risk factors and mortality in patients with first myocardial infarction. JAMA 2011;306:2120-2127.

15. Stamler J, Stamler R, Neaton JD, et al. Low risk-factor profile and long-term cardiovascular and noncardiovascular mortality and life expectancy: findings for 5 large cohorts of young adult and middle-aged men and women. JAMA 1999;282:2012-2018.

16. Aronow WS, Herzig AH, Etienne F, D'Alba P, Ronquillo J. 41-Month follow-up of risk factors correlated with new 
coronary events in 708 elderly patients. J Am Geriatr Soc 1989;37:501-506.

17. Rosa SA, Timoteo AT, Nogueira MA, Belo A, Ferreira RC. Risk factor paradox in the occurrence of cardiac arrest in acute coronary syndrome patients. Rev Bras Ter Intensiva 2016;28:405-412.

18. Ford ES, Ajani UA, Croft JB, et al. Explaining the decrease in U.S. deaths from coronary disease, 1980-2000. N Engl J Med 2007;356:2388-2398.

19. Leifheit-Limson EC, Spertus JA, Reid KJ, et al. Prevalence of traditional cardiac risk factors and secondary prevention among patients hospitalized for acute myocardial infarction (AMI): variation by age, sex, and race. J Womens Health (Larchmt) 2013;22:659-666.
20. Peterson ED, Bynum DZ, Roe MT. Association of evidence-based care processes and outcomes among patients with acute coronary syndromes: performance matters. J Cardiovasc Nurs 2008;23:50-55.

21. Ibanez B, James S, Agewall S, et al. 2017 ESC guidelines for the management of acute myocardial infarction in patients presenting with ST-segment elevation: the Task Force for the management of acute myocardial infarction in patients presenting with ST-segment elevation of the European Society of Cardiology (ESC). Eur Heart J 2018;39:119-177.

22. Marenzi G, Assanelli E, Bartorelli AL. Management of acute coronary syndromes in patients with renal insufficiency. Curr Cardiol Rev 2006;2:11-16. 